\title{
Depressive Störungen bei Jugendlichen: Diagnostik und Therapie
}

\author{
Depressive Disorders in Juveniles: Diagnosis and Therapy
}

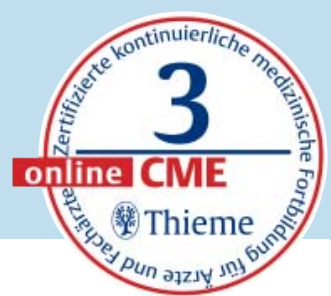

\section{Einleitung \\ $\nabla$}

Depressionen gehören bei Jugendlichen ebenso wie bei Erwachsenen zu den häufigsten und schwerwiegendsten psychischen Störungen und wurden dennoch erst in den letzten Jahren mehr und mehr zu einem relevanten Forschungsthema. Genau das stellt sowohl Theoretiker als auch Praktiker heute vor das große Problem, noch zu wenig über die Entstehung, den Verlauf und v. a. die wirksamsten Therapiemöglichkeiten für diese Altersgruppe $\mathrm{zu}$ wissen. Auswirkungen hat das eher geringe Wissen auch auf die aktuelle Versorgungslage depressiver Jugendlicher, die in Deutschland heute noch als nicht ausreichend anzusehen ist. So erhält derzeit nur etwa die Hälfte der Betroffenen eine nach den Leitlinien der Deutschen Gesellschaft für Kinder- und Jugendpsychiatrie, Psychosomatik und Psychotherapie e.V. (DGKJP) [1] empfohlene Psychotherapie und eine noch geringere Zahl wird mit Psychopharmaka oder einer Kombination aus beiden behandelt.

Die therapeutische Versorgungslage depressiver Jugendlicher ist in Deutschland nicht ausreichend.

Wenn Kinder oder Jugendliche an Depressionen erkranken, hat das häufig gravierende Auswirkungen sowohl auf die psychische als auch auf die physische und soziale Entwicklung der Heranwachsenden. Betroffene zeigen häufiger Schwierigkeiten in sozialen Interaktionen mit Gleichaltrigen, haben mehr Schulprobleme und neigen eher zu Schulabbrüchen. Zusätzlich steigt die Gefahr, Drogen oder Alkohol zu konsumieren und auf die schiefe Bahn zu geraten. Auch die Entwicklung komorbider psychischer Störungen ist im Vergleich zu den Altersgenossen deutlich häufiger und es ist eine höhere Vulnerabilität für somatische Krankheiten zu er- kennen. Zudem steigt mit der bei vielen Jugendlichen zu beobachtenden Isolation und Hoffnungslosigkeit das Risiko einer suizidalen Handlung, sodass Depressionen zu den häufigsten Todesursachen im jungen Erwachsenenalter zählen.

Suizide infolge von Depressionen zählen zu den häufigsten Todesursachen Jugendlicher.

Diese Erkenntnisse zeigen auf, wie wichtig eine Weiterentwicklung der Therapiemöglichkeiten von Depressionen bei Jugendlichen ist.

\section{Epidemiologie und Verlauf \\ $\nabla$}

Depressive Störungen bei Jugendlichen in Europa scheinen immer mehr an Bedeutung zu gewinnen: So stieg die Vergabe der Diagnose Depression zwischen 1995 und 2009 laut dem „The Health Improvement Network (THIN)“ stetig an und auch die Behandlung mit Antidepressiva nahm deutlich zu. Trotzdem gibt es Hinweise darauf, dass die Häufigkeit depressiver Störungen in den letzten Jahren eher stabil geblieben ist [2]. Mögliche Erklärungen für diese kontroversen Befunde sind einerseits eine verbesserte Diagnostik, andererseits ein Überdiagnostizieren in den letzten Jahren. Tab. 1 stellt die aktuellen epidemiologischen Daten für den deutschsprachigen Raum vor.

\section{Die Diagnose „Depression“ bei Jugendlichen \\ $\nabla$}

Wie auch bei Erwachsenen wird eine depressive Störung bei Jugendlichen nach den Kriterien der ICD-10 der WHO [5] diagnostiziert und beinhaltet die folgenden 3 Kernsymptome:
VNR

2760512015147124206

\section{Bibliografie}

Dol http://dx.doi.org/ 10.1055/s-0034-1385776

Fortschr Neurol Psychiatr 2015; 83: 49-62 @ Georg Thieme Verlag KG Stuttgart · New York . ISSN 0720-4299

\section{Erstpublikation}

Dol http://dx.doi.org/ 10.1055/s-0033-1359935 PSYCH up2date 2014; 8: 49-66

Korrespondenzadresse

\section{Dr. Silke Naab}

Fachärztin für Kinderund Jugendpsychiatrie und -psychotherapie, Schön Klinik Roseneck Am Roseneck 6

83209 Prien am Chiemsee SNaab@schoen-kliniken.de 
Tab. 1 Übersicht zu Epidemiologie und Verlauf depressiver Störungen bei Kindern und Jugendlichen.

\begin{tabular}{|c|c|}
\hline Punktprävalenz & $\begin{array}{l}\text { - bei Jugendlichen: } 4-8 \% \\
\text { - } \text { bei Kindern: ca. } 2 \%[3]\end{array}$ \\
\hline Geschlechterverhältnis & $\begin{array}{l}\text { - bei Jugendlichen: } m: w=1: 2 \\
\text { - } \text { bei Kindern: } m: w=1: 1[4]\end{array}$ \\
\hline Risikofaktoren & $\begin{aligned} \text { - } & \text { weibliches Geschlecht } \\
\text { - } & \text { familiäre Häufung } \\
\text { - } & \text { Vorliegen von emotional instabilen } \\
& \text { Persönlichkeitszügen } \\
\text { - } & \text { familiäre Konflikte } \\
\text { - } & \text { negative Lebensereignisse }\end{aligned}$ \\
\hline wichtige Komorbiditäten & $\begin{array}{l}\text { - ca. } 2 / 3 \text { der depressiven Jugendlichen haben mindestens } \\
\text { eine komorbide Störung: } \\
\text { - } \text { Angststörungen/emotionale Störungen }(23,7 \%) \\
\text { - } \text { somatoforme Störungen }(16,8 \%) \\
\text { - hyperkinetische Störung }(16,2 \%) \\
\text { - posttraumatische Belastungsstörung }(10,0 \%) \\
\text { - Verhaltensstörungen }(9,8 \%) \\
\text { - Lese-Rechtschreib-Störung }(8,5 \%)[3]\end{array}$ \\
\hline Verlauf & $\begin{array}{l}\text { - } \quad \text { Dauer einer depressiven Episode: durchschnittlich } \\
\text { - } 8 \text { Monate } \\
\text { - } 50 \% \text { : Remission nach einem Jahr } \\
\text { - } 20 \text { - } 40 \% \text { : Rückfall innerhalb der ersten } 2 \text { Jahre, bis zu } \\
\quad 70 \% \text { : Rückfall innerhalb von } 5 \text { Jahren }\end{array}$ \\
\hline
\end{tabular}

Tab. 2 Depressive Symptome in den verschiedenen Altersstufen [6].

\begin{tabular}{|ll}
$\begin{array}{l}\text { Säuglingsalter } \\
\text { (0-3 Jahre) }\end{array}$ & $\begin{array}{l}\text { Neben Spielunlust und gestörtem Spielverhalten treten häufig } \\
\text { Ess- und Schlafprobleme auf. Der Säugling wirkt irritiert und } \\
\text { traurig und zeigt eine eingeschränkte Mimik und Gestik. }\end{array}$ \\
\hline $\begin{array}{l}\text { frühes Kindesalter } \\
\text { (3-6 Jahre) }\end{array}$ & $\begin{array}{l}\text { Es treten gehäuft Ess- und Schlafprobleme sowie somatische Be- } \\
\text { schwerden (z. B. Kopf- und Bauchschmerzen) auf. Die Kinder wir- } \\
\text { ken traurig, introvertiert und neigen zu aggressiven Ausbrüchen. } \\
\text { Das Spielverhalten ist eingeschränkt und es besteht ein Mangel } \\
\text { an Freude. }\end{array}$ \\
\hline spätes Kindesalter \\
(6-12 Jahre) & $\begin{array}{l}\text { Verschiedene Sorgen und Ängste stehen im Vordergrund. Die } \\
\text { Kinder berichten selbst über eine starke Traurigkeit. Es treten }\end{array}$ \\
& $\begin{array}{l}\text { Schulleistungsstörungen und sozialer Rückzug auf. Zu beachten } \\
\text { sind in diesem Alter mögliche suizidale Gedanken. }\end{array}$ \\
Jugendalter & $\begin{array}{l}\text { Bei Jugendlichen zeigt sich gehäuft selbstverletzendes Verhalten. } \\
\text { (12-19 Jahre) }\end{array}$ \\
$\begin{array}{l}\text { Sie wirken apathisch, interesselos und leiden unter einem gerin- } \\
\text { gen Selbstwertgefühl. In der Folge führt das zu sozialem Rückzug } \\
\text { und Leistungsproblemen in der Schule oder im Beruf. Auch bei } \\
\text { Jugendlichen besteht die Gefahr suizidaler Handlungen. }\end{array}$
\end{tabular}

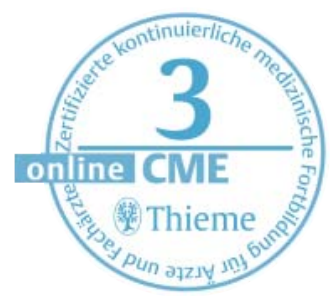

- Antriebslosigkeit

- Verlust der Freude und des Interesses

traurige und niedergedrückte Stimmung

Zusätzlich treten bei Jugendlichen aber auch vermehrt andere Symptome auf, die das Erkennen und Diagnostizieren der Störung in dieser Altersgruppe deutlich erschweren ( $\bullet$ Tab.2). So sollte z.B. beim Vorliegen von chronischen Schmerzen immer auch eine depressive Störung in Betracht gezogen werden.

Erschwert wird die Diagnosestellung durch die bei Jugendlichen häufige Abwehrhaltung gegenüber therapeutischen oder medizinischen Diensten und dadurch, dass viele Jugendliche ihre Erkrankung nicht als solche erkennen [7]. Mit der Dauer der Erkrankung steigt aber die Gefahr einer Chronifizierung und damit auch von möglichen Folgeproblemen, u.a. einer erhöhten Vulnerabilität für psychische Störungen im Erwachsenenalter.
Zur Bewertung des Schweregrads einer Depression bei Jugendlichen ist im Gegensatz zu den Erwachsenen nicht die Anzahl der Symptome ausschlaggebend, sondern es wird das jeweilige Funktionsniveau der Betroffenen herangezogen [8]. Die Symptomatik wird v.a. durch die Exploration des Patienten und seiner Eltern erhoben. Hierbei sollten u. a. die Lebensgeschichte, das erstmalige Auftreten der Symptomatik, mögliche auslösende Faktoren und die Auswirkungen auf den Alltag erfasst werden. Zudem ist eine genaue Erhebung des psychopathologischen Befunds mit Abfrage der Suizidalität unerlässlich.

\section{Tipp für die Praxis}

Bei der Erhebung von Informationen mit einem Kind oder einem Jugendlichen ist sehr auf die Wortwahl und die Art der Fragestellung zu achten: So verneinen viele Jugendliche die Frage nach „Traurigkeit“ und geben eher an, dass sie sich „down“ fühlen.

\section{Diagnostische Testverfahren}

Zur Abklärung depressiver Symptome steht eine Reihe von diagnostischen Testverfahren auch für das Kindes- und Jugendalter zur Verfügung. Neben der Anwendung strukturierter und halbstrukturierter Interviews wie dem „Diagnostischen Interview bei psychischen Störungen im Kindes- und Jugendalter (Kinder-DIPS)“ [9] oder auch projektiven Verfahren (u.a. Wiener Satzergänzungstest nach Rotter, Originalversion [10]) finden in der Praxis v.a. die folgenden störungsspezifischen Selbstbeurteilungsverfahren Anwendung:

- Depressionsinventar für Kinder und Jugendliche (DIKJ) [11]

- Depressionstest für Kinder (DTK) [12]

- Beck-Depressions-Inventar (BDI) [13]

- Children's Depression Rating Scale (CDRS) [14] Als Fremdbeurteilungsverfahren eignet sich v.a. die Hamilton-Skala (Originalversion [15]).

Für eine Diagnosestellung sollten zusätzlich immer fremdanamnestische Daten der Eltern, der Lehrer und anderer Bezugspersonen der Betroffenen eingeholt werden, um ein vollständiges Bild zu erhalten. Auch Verhaltensbeobachtungen haben sich als relevant in der Diagnostik depressiver Störungen bei Kindern und Jugendlichen herausgestellt, wobei v.a. plötzliche Verhaltensänderungen beachtet werden sollten. Zu erwägen ist zudem die Durchführung einer Leistungsdiagnostik mittels üblicher Verfahren (z. B. Hamburg-Wechsler-Intelligenztest für Kinder IV [HAWIK-IV] [16]), um eine möglicherweise symptomauslösende Über- oder Unterforderung in der Schule ausschließen zu können. Bei der Auswertung ist eine Beeinflussung der Testergebnisse durch die depressive Symptomatik zu bedenken. Wichtig ist hier auch die Information 
über einen möglichen Leistungsknick des Patienten in der Vorgeschichte.

\section{Tipp für die Praxis \\ Es muss damit gerechnet werden, dass Jugend- liche bezüglich der Beantwortung der Fragen zur Dissimulation neigen. Daher sind die Tester- gebnisse immer im Zusammenhang mit dem kli- nischen Bild zu interpretieren. Unauffällige Test- ergebnisse schließen das Vorliegen einer Depression nicht aus.}

\section{Differenzialdiagnosen}

Das bei der Depression typische Symptom der Konzentrationsschwäche sowie Appetit- und Schlafprobleme treten auch bei anderen psychischen und organischen Erkrankungen gehäuft auf und müssen daher differenzialdiagnostisch abgeklärt werden. Um organische Ursachen der Symptomatik ausschließen zu können, sollte eine Abklärung gemäß den Leitlinien der DGKJPPP [6] durch eine körperlich-neurologische Routineuntersuchung und eine Labordiagnostik erfolgen. Zum Ausschluss anderer psychischer Störungen wird die Durchführung eines diagnostischen Interviews empfohlen. Folgende psychische Störungen zeigen große Ähnlichkeiten zur depressiven Störung:

- Angststörungen

- bipolare Störung

- Störungen des Sozialverhaltens

- Prodromalstadium einer Schizophrenie

- Aufmerksamkeits- und Hyperaktivitätsstörung

- Substanzmissbrauch

Überblick über die wichtigsten organischen Differenzialdiagnosen

- Hypothyreose

- Anämie

- Nebenwirkungen und/oder Erscheinungsbild verschiedener Medikamente (z. B. Stimulanzien, Kontrazeptiva, Kortisonpräparate)

- Stoffwechselerkrankungen

- Diabetes mellitus

- grippale Infekte

Bipolare Störung. Eine besondere differenzialdiagnostische Bedeutung kommt der bipolaren Störung zu, da diese bei bis zu $40 \%$ der Betroffenen mit einer depressiven Episode beginnt und häufig erstmals im jungen Erwachsenenalter auftritt [17]. Nach einer Studie von Birmaher und Brent [18] haben 20-40\% der Jugendlichen nach der ersten depressiven Episode auch manische und/ oder hypomanische Episoden. Dies hat Auswirkungen auf das therapeutische Vorgehen. So besteht beim Vorliegen oder dem Verdacht einer bipolaren Störung besondere Vorsicht bei einer pharmakologischen Behandlung, da die anti- depressive Medikation eine manische Episode begünstigen oder sogar auslösen kann. Im Gegensatz dazu kann Lithium bei Jugendlichen wirksam zur Phasenprophylaxe bei bipolaren Störungen eingesetzt werden. Zur Abklärung einer bipolaren Störung sollten im Gespräch folgende Risikofaktoren beachtet werden:

- akuter Beginn der depressiven Episode

- Vorhandensein einer bipolaren Störung innerhalb der Familie

Zudem zeichnen sich depressive Episoden innerhalb einer bipolaren Störung häufig durch eine schwere Ausprägung, psychotische Symptome, eine psychomotorische Verlangsamung und intermittierende hypomanische Symptome aus.

\section{Tipp für die Praxis \\ Bei Jugendlichen mit einer bipolaren Störung \\ besteht ein erhöhtes Suizidrisiko!}

\section{Ätiologie}

Wie bei den meisten psychischen Störungen ist auch die Entstehung der Depression bei Jugendlichen auf ein Zusammenspiel aus biologischen, psychologischen und sozialen Faktoren zurückzuführen ( $\bullet$ Abb.1). Bei Jugendlichen konnten v.a. folgende mögliche Risikofaktoren für eine bereits in der Pubertät beginnende Depression identifiziert werden:

- elterliche Vernachlässigung

- problematische soziale Beziehungen zu Gleichaltrigen

- familiäre Probleme

Neben psychosozialen Auslösern spielen aber auch genetische und neurobiologische Faktoren eine wichtige Rolle (u.a. [19]).

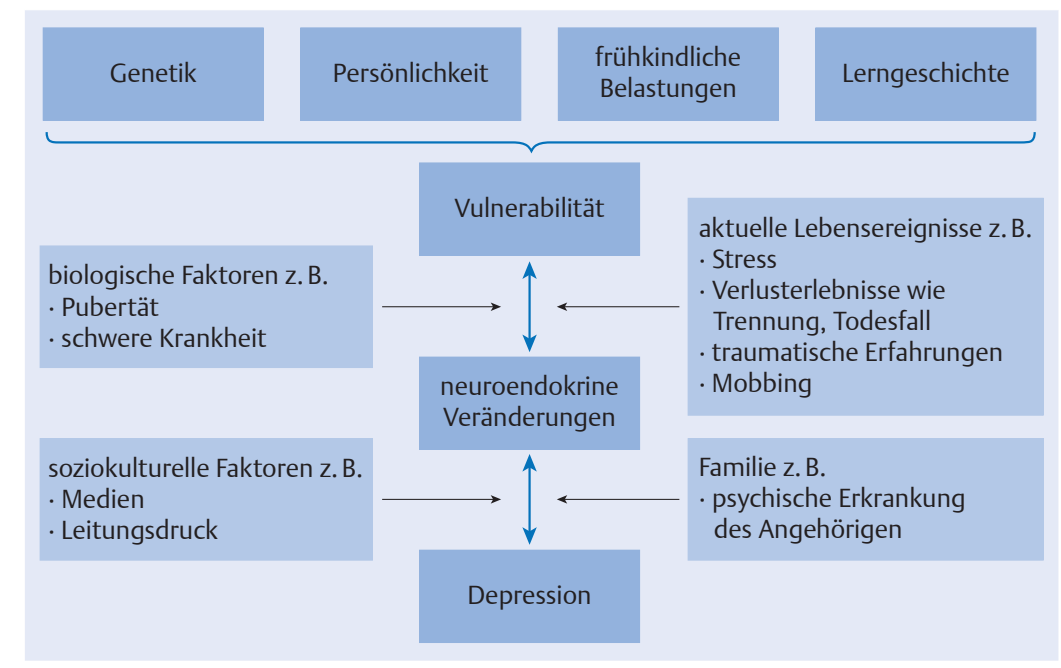

Abb. 1 Multifaktorielles Entstehungsmodell depressiver Störungen bei Jugendlichen. 


\section{Prävention}

$\nabla$

Zur Prävention depressiver Störungen im Kindes- und Jugendalter existiert bereits eine Vielzahl unterschiedlicher Programme, die sowohl universell als auch selektiv und indiziert eingesetzt werden. Besonders häufig finden solche Präventionsprogramme in Gruppen statt und beinhalten dabei v. a. Interventionen aus der kognitiven Verhaltenstherapie. Schulte-Körne und Schiller kommen in ihrer Übersichtsarbeit, in die insgesamt 121 Studien einbezogen wurden, zu dem abschließenden Ergebnis, dass der Einsatz von Präventionsprogrammen bei Jugendlichen im Gegensatz zu einer nicht behandelten Kontrollgruppe zu einer Reduktion der depressiven Symptome führt [20]. Diese Erfolge konnten für alle Programme sowohl kurzfristig als auch in einem Zeitraum von bis zu 9 Monaten gezeigt werden. Nach 12 Monaten scheinen nur noch die selektiven Präventionsprogramme wirksam zu sein. Einschränkend ist jedoch zu sagen, dass die einbezogenen Studien sehr heterogen und auch die erreichten Effektstärken insgesamt als niedrig zu bewerten sind.

\section{Gruppentherapeutische \\ Ansätze an Schulen}

Gilham und Kollegen evaluierten ein gruppentherapeutisches Präventionsprogramm, das von Lehrern an einer Schule durchgeführt wurde [21]. Die Schüler in der Altersgruppe von 10-15 Jahren wurden randomisiert einer von 3 Bedingungen zugeordnet:

- Teilnahme am Gruppenprogramm

- Teilnahme am Gruppenprogramm und gleichzeitiges Gruppenprogramm für die Eltern

- Teilnahme am normalen Unterricht

Insgesamt zeigten die Schüler, die am Präventionsprogramm teilnahmen, sowohl direkt nach dem Programm als auch nach 6 Monaten reduzierte Angst- und Depressionswerte, einen verbesserten Coping-Stil und Veränderungen in den Kognitionen im Vergleich zu den Schülern mit normalem Unterricht. Das zusätzliche Gruppentraining der Eltern ergab keine darüber hinausgehenden Effekte.

Horowitz und Kollegen verglichen in ihrer Studie die Wirksamkeit eines kognitiv-verhaltenstherapeutischen mit der eines interpersonell-fertigkeitsorientierten Präventionsprogrammes [22]. Dabei zeigten sich beide Programme einer unbe-

Tab. 3 Präventionsprogramm LARS \& LISA [25].

\begin{tabular}{|c|c|}
\hline Aufbau & Inhalte der Sitzungen \\
\hline kognitive Sitzungen (5 Stunden) & $\begin{array}{l}\text { Zusammenhang zwischen Kognitionen, Emotionen } \\
\text { und Verhalten } \\
\text { Identifizierung und Modifikation negativer Gedanken }\end{array}$ \\
\hline soziale Sitzungen (4 Stunden) & $\begin{array}{l}\text { Selbstbehauptungstraining } \\
\text { Training sozialer Kompetenzen }\end{array}$ \\
\hline motivationale Sitzung (1 Stunde) & individuelle Zielsetzung und Zielerreichung \\
\hline
\end{tabular}

handelten Kontrollgruppe gegenüber als überlegen; Unterschiede zwischen den beiden konnten dagegen nicht gefunden werden.

Ein gruppentherapeutisches Präventionsprogramm in der Schule ist wirksam zur Reduktion von Angst- und Depressionswerten.

In einer groß angelegten Studie evaluierten Pössel, Adelson und Hautzinger das als Manual vorliegende, gruppentherapeutische Präventionsprogramm LARS \& LISA, das 2004 in Deutschland erarbeitet wurde [23]. Das Programm umfasst 10 Sitzungen zu je 90 Minuten und wird an Schulen durch ausgebildete Therapeuten durchgeführt ( $\bullet$ Tab.3). Es zeigte sich, dass depressive Symptome bei Mädchen in den ersten 6 Monaten nach Ende des Trainings abnahmen und dann in den folgenden Monaten stabil blieben, während bei Jungen keine positiven Effekte nachgewiesen werden konnten. Eine schlüssige Erklärung dieser Geschlechterunterschiede konnte derzeit noch nicht gefunden werden; die Differenzen sind jedoch nicht alleine durch kognitive oder soziale Unterschiede zu erklären.

Im Gegensatz dazu fanden Stallard und Kollegen bei ihrer Evaluation eines präventiven Gruppenprogrammes an Schulen keine Unterschiede in den Depressionswerten von Kindern mit einem hohen Depressionsrisiko zwischen der Experimentalgruppe (Teilnahme am Gruppentraining) und den Kontrollgruppen (Training zur Aufmerksamkeitskontrolle oder Teilnahme am normalen Unterricht) [24].

Kritische Zusammenfassung. Trotz der hohen Relevanz von Präventionsarbeit zur Vermeidung depressiver Störungen bei Jugendlichen ist die Studienlage über die Wirksamkeit derzeit noch sehr heterogen und zu viele Studien weisen methodologische Mängel auf [26]. Trotzdem zeigen einige placebokontrollierte Studien gute Effekte in der Reduktion von depressiven Symptomen nach der Teilnahme an kognitiv-verhaltenstherapeutischen und interpersonellen Präventionsprogrammen, sodass weitere wissenschaftliche Untersuchungen auf diesem Gebiet wünschenswert sind. Auch die Persistenz der Ergebnisse spricht für einen über den Einsatz von Placebos hinausgehenden positiven Effekt [27]. Nach Merry sollte der Fokus in der nächsten Zeit v. a. darauf gelegt werden, dass nicht mehr depressive Symptome, sondern depressive Episoden als relevantes Outcome-Maß herangezogen werden und dass ein genaueres Augenmerk auf die eigentlich wirksamen Interventionen gelegt wird [26]. 


\section{Fazit für die Praxis}

Sowohl universelle und selektive als auch indizierte Präventionsprogramme zeigen in einigen Studien eine deutliche Reduktion depressiver Symptome. Je früher die Problematik erkannt wird, desto besser sind die Heilungschancen einzuschätzen.

\section{Psychotherapie}

Psychotherapie gilt nach einer Studie von Caporino und Karver als die von Jugendlichen am besten akzeptierte Therapieform, wobei die Art der therapeutischen Intervention von der aktuellen sozialen Situation und den möglichen Auslösebedingungen abhängig ist [28]. Auch gemäß den Leitlinien der DGKJP [1] stellen psychotherapeutische Interventionen die Therapie der 1. Wahl dar und sollten nur dann mit einer pharmakologischen Behandlung kombiniert werden, wenn

- die Jugendlichen nicht oder zu wenig auf die durchgeführten Interventionen reagieren,

- schwere depressive Störungen und/oder komorbide Störungen vorliegen, die den Therapieverlauf negativ beeinflussen oder

- es aufgrund mangelnder Therapieplätze zu einer Wartezeit kommt.

Gute Erfolge der Psychotherapie werden v. a. bei Jugendlichen mit leichten bis mittelschweren Verläufen erzielt.

Die Behandlung erfolgt - wenn möglich - in einem ambulanten Setting, um die Auswirkungen auf das soziale Leben der Jugendlichen möglichst gering zu halten. Neben der Schwere der Depression und dem Vorliegen komorbider Störungen sollten weitere Faktoren bei einer Entscheidung für eine stationäre Therapie berücksichtigt werden.

Kriterien für die Aufnahme einer stationären
Therapie
Vorliegen von Suizidideen oder suizidalen
Tendenzen
schwierige familiäre Verhältnisse (z. B. Miss-
brauch, Misshandlung, Konflikte)
Schulschwierigkeiten oder schwerwiegende
Probleme im sozialen Kontakt mit Gleich-
altrigen
erfolglose ambulante Psychotherapie

\section{Tipp für die Praxis}

Notwendige teilstationäre oder stationäre Therapien sollten gemäß den Leitlinien [1] frühzeitig erfolgen und bedürfen immer eines Gesprächs mit den Betroffenen und den Eltern.

Vorgehen. Das Vorgehen in der Therapie muss immer vom jeweiligen Schweregrad der depressiven Episode und dem vorliegenden psychosozialen Funktionsniveau anhängig gemacht werden: Bei leichten Depressionen können in einem ersten Schritt kurze Beratungen und Psychoedukation eingesetzt werden, während bei einer mittelgradigen Depression immer der Beginn einer Psychotherapie empfohlen wird. Diese sollte bei schweren Depressionen durch eine pharmakologische Behandlung ergänzt werden (s. o.).

Die höchste Effektivität psychotherapeutischer Verfahren konnte bisher bei Studien mit kognitiv-verhaltenstherapeutischen Interventionen erreicht werden.

Im Gegensatz zu der nachgewiesenen Wirksamkeit von einzel- und gruppentherapeutischen Verfahren ist die Effektivität eines Einbezugs der Eltern in die Therapie noch immer nicht eindeutig geklärt. Gemäß den Leitlinien [6] sollten die Eltern zu Beginn der Therapie zur Vervollständigung der Anamnese herangezogen werden, v.a. um mögliche Dispositionen, familiäre Belastungen und die aktuelle Befindlichkeit des Jugendlichen abzuklären. Inwieweit Eltern auch den Verlauf der Therapie positiv beeinflussen können, ist unklar.

\section{Tipp für die Praxis \\ Neben dem Schweregrad der Störung findet in der Wahl der geeigneten Therapie auch immer das Alter und der jeweilige Entwicklungsstand der Betroffenen Berücksichtigung: Jugendliche profi- tieren neben einer Kognitiven Verhaltenstherapie auch von einer interpersonellen Einzeltherapie.}

\section{Kognitive Verhaltenstherapie}

Die Kognitive Verhaltenstherapie (KVT) gilt gemäß den Leitlinien der DGKJP [1] als Therapie erster Wahl; ihre Wirksamkeit wird durch die Ergebnisse vieler Studien bewiesen (Übersicht [29]). Vor allem in der Akutphase zeigt sich diese Therapieform gegenüber Wartelistenkontrollgruppen und auch anderen Psychotherapien überlegen [30]. Für die Wirksamkeit im Langzeitverlauf liegen derzeit jedoch noch keine eindeutigen Beweise vor: Erford und Kollegen zeigten in ihrer Metaanalyse anhaltende Effekte bis

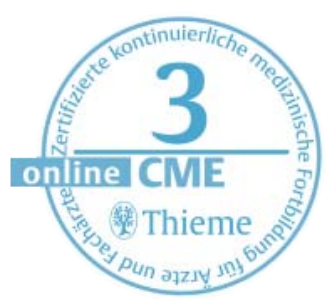


Tab. $4 \mathrm{MICHI}$ - Aufbau und Inhalte des gruppentherapeutischen Programmes.

\begin{tabular}{|l|l|}
\hline Sitzungen & Inhalte \\
\hline $\begin{array}{l}\text { 1. Mehr Wissen über meine } \\
\text { Depression }\end{array}$ & - Kennenlernen der Gruppe \\
\hline 2. Die Sache mit den Gefühlen & - Psychoedukation \\
& - Undividuelle Ressourcen und Stärken \\
\hline $\begin{array}{l}\text { 3. Ich kann meine Gefühle } \\
\text { beeinflussen }\end{array}$ & - Selbstwert \\
\hline $\begin{array}{l}\text { 4. Ich kann Gedanken verändern } \\
\text { 5. Gesund bleiben mit Unter- }\end{array}$ & - Gedanken kontrollieren und verändern lernen \\
stützung anderer & - Umgang mit Krisen \\
\hline $\begin{array}{l}\text { 6. Auffrischungssitzung } \\
\text { (nach } 5 \text { Wochen) }\end{array}$ & - Einbezug von Vertrauenspersonen: Psychoedu- \\
\hline
\end{tabular}

Tab. 5 Übersicht über Therapieverfahren bei Jugendlichen mit Depressionen und deren Evidenzgrad gemäß den Leitlinien der DGKJP [1].

\begin{tabular}{|c|c|c|}
\hline Therapie $^{1}$ & Therapeutisches Vorgehen & Evidenz ${ }^{2}$ \\
\hline KVT & $\begin{array}{l}\text { kognitive Techniken (Methoden der kognitiven Umstruktu- } \\
\text { rierung, Selbstinstruktionstraining, Problemlösetraining, } \\
\text { Selbstmanagement) } \\
\text { familienbezogene Techniken (Kommunikationstraining, } \\
\text { Elterntraining) } \\
\text { verhaltensorientierte Techniken (Aktivitätsaufbau, Selbst- } \\
\text { und Fremdverstärkung, soziales Kompetenztraining) } \\
\text { emotionsbezogene Techniken (Training der Emotionserken- } \\
\text { nung, Ärgerkontrolltraining, Entspannungsverfahren) [6] }\end{array}$ & I \\
\hline IPT & $\begin{array}{l}\text { kurzes, pragmatisches und alltagsnahes Vorgehen als Kom- } \\
\text { bination zwischen psychoanalytischen und kognitiv-verhal- } \\
\text { tenstherapeutischen Konzepten } \\
\text { Bausteine: } \\
\text { Umgang mit pathologischer Trauer } \\
\text { Reduktion interpersoneller Konflikte und Defizite } \\
\text { Umgang mit Rollenwechseln [37] }\end{array}$ & II \\
\hline FAM & $\begin{array}{l}\text { Bearbeitung von Defiziten und Dysfunktionen in der fami- } \\
\text { liären Kommunikation und von Beziehungskonflikten } \\
\text { Ziele: } \\
\text { Wiederherstellung einer tragfähigen und vertrauensvollen } \\
\text { Beziehung innerhalb der Familie } \\
\text { Stärkung der Eltern in ihrem Erziehungsverhalten } \\
\text { Einsatz v. a. bei jüngeren Kindern und Familien, die das } \\
\text { Auftreten der depressiven Störung des Jugendlichen als } \\
\text { familiäres Problem definieren [38] }\end{array}$ & III-IV \\
\hline TP & $\begin{array}{l}\text { Bearbeitung der zugrunde liegenden Konflikte und proble- } \\
\text { matischen Objektbeziehungen in einer vertrauensvollen } \\
\text { therapeutischen Beziehung } \\
\text { Einsatz von kunst- und spieltherapeutischen Elementen }\end{array}$ & III-IV \\
\hline \multicolumn{3}{|c|}{$\begin{array}{l}\text { I KVT: Kognitive Verhaltenstherapie; IPT: Interpersonale Psychotherapie; FAM: Familientherapie; TP: } \\
\text { tiefenpsychologisch orientierte Therapie } \\
\text { 2 Evidenzklassen: } \\
\text { I: Evidenz durch wenigstens eine Metaanalyse auf Basis methodisch hochwertiger, randomisierter kon- } \\
\text { trollierter Studien; II: Evidenz von mindestens einer gut angelegten (hochwertigen), jedoch nicht rando- } \\
\text { misierten und kontrollierten Studie; III: Evidenz aufgrund gut angelegter (methodisch hochwertiger), } \\
\text { nichtexperimenteller deskriptiver Studien wie etwa Vergleichsstudien, Korrelationsstudien oder Fall-Kon- } \\
\text { troll-Studien; IV: Evidenz aufgrund der Berichte von Expertenausschüssen oder Expertenmeinungen bzw. } \\
\text { klinischer Erfahrung (Meinungen und Überzeugungen) anerkannter Autoritäten; beschreibende Studien; } \\
\text { V: Fallserie oder eine/mehrere Expertenmeinungen. }\end{array}$} \\
\hline
\end{tabular}

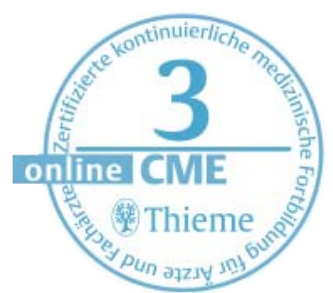

zu 24 Monate; nach einem längeren Zeitraum konnten dagegen kaum noch positive Effekte nachgewiesen werden [31].
Neben dem Aufbau einer vertrauensvollen therapeutischen Beziehung sollte der Fokus in der Behandlung von Jugendlichen v. a. auf kognitiven Techniken liegen.

Neben einer einzeltherapeutischen Behandlung zeigen sich auch gruppentherapeutische Ansätze in der Therapie von Jugendlichen als effektive Methode. Ein von Abel evaluiertes kognitiv-verhaltenstherapeutisches Gruppentherapieprogramm zeigte bei den 12- bis 18-jährigen Teilnehmern eine deutliche Reduktion der depressiven Symptomatik sowohl in der Selbstbeurteilung der Betroffenen als auch in der Einschätzung der Eltern [32]. Ströber und Kollegen entwickelten ein spezielles kognitiv-behaviorales Gruppentherapieprogramm (MICHI - Manualized Intervention to Cope with depressive symptoms, Help to strengthen ressources and Improve emotion regulation) zur Behandlung von depressiven Jugendlichen, dessen Wirksamkeit in einer randomisierten kontrollierten Studie nachgewiesen werden konnte [33][34]. Das Therapieprogramm kann sowohl stationär als auch ambulant eingesetzt werden und umfasst insgesamt 6 Sitzungen zu je 75 Minuten ( $\bullet$ Tab. 4), wobei die Inhalte altersentsprechend vermittelt werden.

\section{Andere psychotherapeutische Verfahren}

Eine Übersicht über die verschiedenen Therapieverfahren ist in $\bullet$ Tab. 5 dargestellt.

Interpersonale Psychotherapie. Neben der KVT konnte in Studien auch eine Effektivität der Interpersonalen Psychotherapie (IPT) in der Akutbehandlung von Depressionen bei Jugendlichen gezeigt werden: Mufson und Kollegen wiesen nach einer 12-wöchigen interpersonellen Therapie eine Reduktion der depressiven Symptome und eine Steigerung der sozialen Leistungsfähigkeit sowie der Problemlösefertigkeiten nach [35]. Auch Curry fand in 2 Studien eine Überlegenheit der IPT gegenüber einer Wartelistenkontrollgruppe und einer 2-stündigen Beratung [30].

Familientherapie. Die Familientherapie konnte bisher noch keine überzeugenden Nachweise ihrer Wirksamkeit erbringen. Auch die Ergänzung familientherapeutischer Interventionen zu einer KVT scheint nicht zu einem besseren Ergebnis zu führen [1].

Tiefenpsychologische Verfahren. Zum Einsatz tiefenpsychologischer oder psychoanalytischer Interventionen liegen derzeit noch keine methodisch hochwertigen und kontrollierten Studien vor, sodass bislang keine Aussagen zur Wirksamkeit möglich sind.

Lichttherapie. Einzelne Studien zeigen positive Effekte in der Reduktion von saisonalen Depressionen durch die Behandlung mit Lichttherapie [36], wobei die Ergebnisse aufgrund der geringen Datenlage mit Vorsicht zu interpretieren sind. 


\section{Computerisierte Selbsthilfeprogramme}

Trotz der Schwere der Erkrankung und den belastenden psychosozialen Auswirkungen haben viele Jugendliche Probleme, sich auf eine konventionelle Psychotherapie einzulassen oder sich überhaupt Hilfe und Unterstützung zu suchen. Daher werden immer häufiger computerisierte Selbsthilfeprogramme auch für Jugendliche entwickelt. Diese haben sich schon in der Behandlung von depressiven Erwachsenen als wirksam erwiesen [39]. Vorteile der computerisierten Programme sind neben der guten Verfügbarkeit auch die zeitliche und räumliche Flexibilität in der Nutzung und die im Vergleich zur Psychotherapie niedrigeren Kosten. Die Wirksamkeitsnachweise von computerisierten Verfahren sind allerdings derzeit noch sehr heterogen und daher können diese Programme nicht als möglicher Ersatz einer herkömmlichen Psychotherapie angesehen werden.

- Tab. 6 stellt 2 evaluierte Therapieprogramme vor:

- SPARX

PST

\section{Kombinationstherapie}

Gemäß den Leitlinien der DGKJP ist beim Vorliegen einer schweren depressiven Episode eine Kombination aus psychotherapeutischer und psychopharmakologischer Therapie indiziert [1]. Trotz dieser Empfehlungen ist die Studienlage zur Wirksamkeit der Kombinationsbehandlung noch sehr heterogen: Die groß angelegte Treatment-ofAdolescent-with-Depression-Study kam zu dem Ergebnis, dass eine Kombination aus KVT und dem selektiven Serotonin-Wiederaufnahmehemmer (SSRI) Fluoxetin zwar zu einer schnelleren Abnahme der depressiven Symptome führte; am Ende der Behandlung bestanden aber keine Vorteile gegenüber einer pharmakologischen Monotherapie [41]. Cox und Kollegen zeigten in ihrer umfassenden Metaanalyse ein ähnliches Bild [42]: In den meisten Studien wurden keine Unterschiede in der Wirksamkeit zwischen Psychotherapie, Psychopharmakotherapie und einer Kombinationsbehandlung gefunden. Nur 2 Studien mit 220 Teilnehmern wiesen auf eine Überlegenheit der pharmakologischen Monotherapie hin, die sich v.a. in den Remissionsraten nach Behandlungsende zeigte. Drei Studien zeigten eine höhere Effektivität einer Kombinationsbehandlung gegenüber einer Psychopharmakotherapie; gegenüber einer alleinigen Psychotherapie konnte keine Studie eine Überlegenheit nachweisen. Nach Curry und Kollegen wirkt die Kombinationsbehandlung v.a. bei leichten und mittelgradigen Depressionen, wohingegen bei schweren Depressionen eine medikamentöse Therapie überlegen ist [43].
Tab. 6 Computerisierte Selbsthilfeprogramme.

\begin{tabular}{|c|c|c|}
\hline Programm $^{1}$ & Inhalte & Wirksamkeit \\
\hline SPARX [26] & $\begin{array}{l}\text { - } \text { kognitiv-verhaltenstherapeuti- } \\
\text { sches Programm mit } 7 \text { Modulen } \\
\text { - } \text { Aufbau als interaktives Compu- } \\
\text { terspiel, in dem die Betroffenen } \\
\text { sowohl als eigene Person in Kon- } \\
\text { takt mit einem Experten treten } \\
\text { als auch als Spielfigur Aufgaben } \\
\text { lösen müssen }\end{array}$ & $\begin{array}{l}\text { Reduktion der Depressionswer- } \\
\text { te und höhere Remissionsrate } \\
\text { im Vergleich zu „Treatment as } \\
\text { usual“ bei schwereren Depres- } \\
\text { sionen [1] }\end{array}$ \\
\hline PST [40] & $\begin{array}{l}\text { - kognitiv-verhaltenstherapeuti- } \\
\text { sches Problemlösetraining über } \\
5 \text { Wochen } \\
\text { - } \text { Ablauf: Teilnehmer erhalten per } \\
\text { E-Mail eine Wochenaufgabe und } \\
\text { bekommen im Anschluss Feed- } \\
\text { back eines Experten }\end{array}$ & $\begin{array}{l}\text { keine Überlegenheit der PST } \\
\text { gegenüber einer Wartelisten- } \\
\text { kontrollgruppe }\end{array}$ \\
\hline
\end{tabular}

1 SPARX: Smart, Positive, Active, Realistic, X-factor Thoughts; PST: Problem Solving Therapy.

Tipp für die Praxis
Laut den Leitlinien der DGKJP ist die Psychothera-
pie in der Behandlung depressiver Störungen die
Therapie der Wahl [1]. Bei schweren Verläufen
empfiehlt sich eine begleitende pharmakologi-
sche Behandlung.

\section{Psychopharmakotherapie}

Der Einsatz von Psychopharmaka stellt in der Behandlung v.a. von schweren Depressionen bei Kindern und Jugendlichen eine mögliche Therapieoption dar. Eine Metaanalyse von Hetrick und Kollegen zeigte eine Überlegenheit von Antidepressiva gegenüber Placebo, auch wenn die erreichten Effektstärken als niedrig einzuschätzen sind [44]. Sowohl die deutschen [28] als auch die amerikanischen [45] und britischen [46] Leitlinien der Fachgesellschaften empfehlen den Einsatz einer antidepressiven Medikation immer in Ergänzung zu einer Psychotherapie, bzw. wenn eine anfängliche Psychotherapie nicht zu einer Verbesserung der depressiven Symptomatik führte. Nach Plener, Fegert und Kölch [47] hat sich in Deutschland nach der Debatte um die Gefahren einer antidepressiven Medikation bei Jugendlichen die Versorgungslage auf diesem Gebiet deutlich verbessert, auch wenn immer noch nicht alle Kinder und Jugendliche die nach aktuellem Wissensstand bestmögliche Behandlung bekommen [2]. So erhalten nach einer Auswertung der Versichertenstände aus dem Jahr 2009 noch immer $17,9 \%$ der Jugendlichen eine Behandlung mit einem trizyklischen Antidepressivum und 8,5\% werden mit Johanniskraut behandelt. Trotzdem ist die Zahl der eingesetzten SSRI in den Jahren zwischen 2000 und 2009 von $15 \%$ auf 55,6\% gestiegen [48].

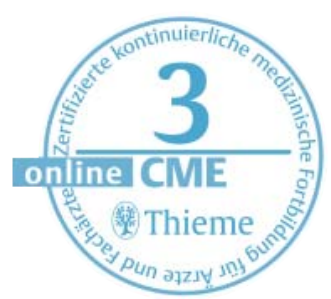


Fluoxetin ist das einzige in Europa zugelassene antidepressive Medikament für Kinder ab 8 Jahren und wird bei mittelgradigen und schweren depressiven Störungen empfohlen [6].

Die Mehrzahl der antidepressiven Medikation bei Kindern und Jugendlichen erfolgt im Rahmen der Off-Label-Verordnung, worüber sowohl die Betroffenen als auch die Eltern ausführlich aufgeklärt werden müssen.

Tipp für die Praxis

Vor dem Beginn einer pharmakologischen Behandlung sollten eine Labordiagnostik sowie ein Elektrokardiogramm erfolgen.

\section{Tipp für die Praxis}

Bei Kindern und Jugendlichen werden depressive Episoden häufig reaktiv ausgelöst, sodass unter diesen Bedingungen auch schwere Verläufe besser auf eine Psychotherapie ansprechen als bei Erwachsenen. So zeigten Goodyer und Kollegen in ihrer Studie, dass $21 \%$ der Jugendlichen auch mit mittelgradigen und schweren Depressionen von einer alleinigen psychotherapeutischen Behandlung profitierten [49].

\section{Selektive Serotonin-}

Wiederaufnahmehemmer

Nach aktuellem Stand der Forschung sind Fluoxetin [50], Citalopram [51] und Sertralin [52] derzeit die einzigen SSRI, die in verschiedenen, placebokontrollierten Studien bei Depression im Jugendalter ihre Überlegenheit beweisen konnten und auch das niedrigste relative Suizidrisiko aufweisen [53].

\section{Tipp für die Praxis}

Fluoxetin kann bei Jugendlichen in einer Dosis von $20 \mathrm{mg} / \mathrm{d}$ eingesetzt werden. Aufgrund der hohen Halbwertszeit von ca. 46 Stunden (aktiver Metabolit noch länger) ist eine Einmalgabe pro Tag zu empfehlen.

Weniger eindeutig ist die Datenlage in Bezug auf Paroxetin, zu dem bisher keine methodisch hochwertigen, kontrollierten Wirksamkeitsnachweise bei Jugendlichen vorliegen [54].

Ansprechraten. Insgesamt wird bei Kindern und Jugendlichen von einer Responserate auf Antidepressiva zwischen 40 und $70 \%$ ausgegangen. Bei einem Nichtansprechen kann ein Wechsel auf ein anderes SSRI erwogen werden, da sich diese in ihrer Wirkungsweise und auch dem Nebenwirkungsprofil unterscheiden; diese Empfehlung stützt sich aber (wie auch bei Erwachsenen) nicht auf Studien. Nach einer Remission der depressiven Symptomatik empfiehlt sich die Weitergabe über 6 Monate, sodass einem möglichen Rückfall vorgebeugt werden kann.

Therapieresistenz. Beim Vorliegen von therapieresistenten Depressionen wurden in der TORDIA-Studie (TORDIA: Treatment of resistant Depression in Adolescents) Versuche unternommen, eine pharmakologische Monotherapie entweder durch die Aufnahme einer KVT oder durch die zusätzliche Einnahme von Venlafaxin zu ergänzen [55]. Es zeigten sich daraufhin keine Verbesserungen gegenüber der Einnahme eines einzelnen Antidepressivums; im Gegensatz dazu nahmen unter einer gleichzeitigen Einnahme eines SSRI mit Venlafaxin die unerwünschten Nebenwirkungen und auch die Suizidideen zu. Im Unterschied zu Erwachsenen liegen sonst für das Kindes- und Jugendalter nur wenige Studien zu einer medikamentösen Augmentation vor: Erste Untersuchungen zeigen aber eine mögliche Verbesserung der Wirksamkeit von SSRI bei therapieresistenter Depression durch Bupropion [56] und dem atypischen Neuroleptikum Quetiapin [57].

\section{Andere Psychopharmaka}

Trizyklische Antidepressiva. Trotz der nachgewiesenen Wirksamkeit der SSRI werden in der Praxis Kinder und Jugendliche auch heute immer wieder mit anderen Substanzklassen behandelt. Am häufigsten ist dabei die Verschreibung von trizyklischen Antidepressiva (TZA): Die Ergebnisse einer Metaanalyse von Hazell und Kollegen ergaben jedoch nur eine moderate Wirkung der TZA in der Behandlung von Jugendlichen [58]. Auch die Leitlinien der DGKJP weisen darauf hin, dass TZA in bisherigen Studien keine Überlegenheit gegenüber Placebo aufwiesen und daher in der Behandlung dieser Altersgruppe nicht zu empfehlen sind [1].

SNRI. Zur Wirksamkeit von selektiven SerotoninNoradrenalin-Wiederaufnahmehemmern (SNRI) liegen derzeit noch wenige Studien vor: Zwei Studien untersuchten die Wirksamkeit von Venlafaxin und konnten keine Überlegenheit gegenüber Placebo nachweisen [59, 60]. Das SNRI Duloxetin zeigte in einer Studie von Prakash und Kollegen eine gute Toleranz bei Kindern und Jugendlichen, es traten aber verstärkt kardiale Nebenwirkungen auf und die Wirksamkeit gegenüber Placebo wurde nicht untersucht [61].

Johanniskraut. Das pflanzliche Arzneimittel Johanniskraut (Hypericum-Extrakt) wird in Deutschland neben SSRI und TZA bei Kindern und Jugendlichen am häufigsten zur Behandlung von Depressionen eingesetzt, auch wenn notwendige kontrollierte Wirksamkeitsnachweise bisher noch fehlen [54]. Trotzdem ist das Präparat bei Kindern ab 12 Jahren zugelassen.

Eine ergänzende Übersicht zu den psychopharmakologischen Behandlungsmöglichkeiten gibt

- Tab. 7. 


\begin{tabular}{|c|c|c|c|}
\hline Substanzklasse & Nebenwirkungen & Empfehlung & Evidenzgrad $^{1}$ \\
\hline SSRI & $\begin{array}{l}\text { Müdigkeit, Schlafprobleme, } \\
\text { Erbrechen, sexuelle } \\
\text { Dysfunktion }\end{array}$ & $\begin{array}{l}\text { - Substanz der 1. Wahl } \\
\text { (v. a. Fluoxetin) } \\
\text { - SSRI können in der An- } \\
\text { fangsphase suizidale Ge- } \\
\text { danken verstärken! }\end{array}$ & 1 \\
\hline TZA & $\begin{array}{l}\text { kardiale Probleme, Mundtro- } \\
\text { ckenheit, Tremor, Schwindel, } \\
\text { Zittern }\end{array}$ & $\begin{array}{l}\text { - } \text { keine Effekte bei Kindern; } \\
\text { bei Jugendlichen moderate } \\
\text { Effekte } \\
\text { - } \\
\text { Nachteil: geringe thera- } \\
\text { peutische Breite }\end{array}$ & $\begin{array}{l}\text { keine Wirk- } \\
\text { samkeit: I }\end{array}$ \\
\hline $\begin{array}{l}\text { MAO-Hemmer } \\
\text { (Monoaminoxidasehemmer) }\end{array}$ & $\begin{array}{l}\text { Bewegungsstörungen, } \\
\text { Schwindel, Unruhe, Blut- } \\
\text { druckkrisen, Kopfschmerzen }\end{array}$ & $\begin{array}{l}\text { - große Vorsicht wegen star- } \\
\text { ken Nebenwirkungen und } \\
\text { Erhöhung der Suizidalität }\end{array}$ & $\begin{array}{l}\text { keine Wirk- } \\
\text { samkeit: III }\end{array}$ \\
\hline $\begin{array}{l}\text { Phasenprophylaxe (Lithium; } \\
\text { Carbamazepin) }\end{array}$ & $\begin{array}{l}\text { u. a. vermehrtes Durstgefühl/ } \\
\text { Wasserlassen, Müdigkeit, Zit- } \\
\text { tern, Gewichtszunahme }\end{array}$ & $\begin{array}{l}\text { - Einsatz bei schweren rezi- } \\
\text { divierenden Verläufen } \\
\text { möglich } \\
\text { - wirksam zur Phasenpro- } \\
\text { phylaxe bei bipolaren } \\
\text { Störungen }\end{array}$ & IV - V \\
\hline Johanniskraut & $\begin{array}{l}\text { Fotosensibilisierung, Induk- } \\
\text { tion von Zytochrom CYP3A4 }\end{array}$ & $\begin{array}{l}\text { - } \text { bei leichten bis mittel- } \\
\text { gradigen Depressionen } \\
\text { einsetzbar } \\
\text { - } \text { gute Verträglichkeit }\end{array}$ & IV \\
\hline
\end{tabular}

Tab. 7 Übersicht zur psychopharmakologischen Behandlung von Depressionen bei Jugendlichen.

I: Evidenz durch wenigstens eine Metaanalyse auf Basis methodisch hochwertiger, randomisierter kontrollierter Studien; II: Evidenz von mindestens einer gut angelegten (hochwertigen), jedoch nicht randomisierten und kontrollierten Studie; III: Evidenz aufgrund gut angelegter (methodisch hochwertiger), nichtexperimenteller deskriptiver Studien wie etwa Vergleichsstudien, Korrelationsstudien oder Fall-Kontroll-Studien; IV: Evidenz aufgrund der Berichte von Expertenausschüssen oder Expertenmeinungen bzw. klinischer Erfahrung (Meinungen und Überzeugungen) anerkannter Autoritäten; beschreibende Studien; V: Fallserie oder eine/mehrere Expertenmeinungen.

\section{Nicht-pharmakologische somatische Verfahren bei therapieresistenten Depressionen \\ $\nabla$}

In der Behandlung von Depressionen bei Jugendlichen, v.a. bei schweren Formen oder bei einer Therapieresistenz, wird neben dem Einsatz von psychotherapeutischen und pharmakologischen Therapien auch der Einsatz und die Wirksamkeit anderer somatischer Verfahren diskutiert.

Elektrokrampftherapie. Die Elektrokrampftherapie (EKT) basiert auf durch Strom unter Kurznarkose ausgelöste Krampfanfälle. [62]. Während dieses invasive Verfahren bei Erwachsenen seit Langem als wirksam in der Behandlung therapieresistenter Depressionen etabliert ist [63], liegen aufgrund der Schwere des Eingriffs und der möglichen unerwünschten Nebenwirkungen (u. a. Kopf- und Muskelschmerzen, Ausfälle des Kurzzeitgedächtnisses) nur wenige Studien mit Jugendlichen vor. Strober und Kollegen zeigten jedoch auch bei Jugendlichen mit therapieresistenter Depression Verbesserungen in der depressiven Symptomatik [64]. Gemäß den Leitlinien der American Academy of Child and Adolescent Psychiatry (AACAP) [65] kann eine Behandlung mit EKT nach 2 oder mehr fehlgeschlagenen pharmakologischen Versuchen auch bei Jugendlichen angedacht werden, wobei immer die Zustimmung sowohl der Betroffenen als auch der Eltern eingeholt werden muss.

Repetitive transkranielle Magnetstimulation. Als weiteres nichtpharmakologisches somatisches Verfahren wurde, ebenso wie bei Erwachsenen [66], die repetitive transkranielle Magnetstimulation (rTMS) eingesetzt. In der bisher größten Studie mit Jugendlichen zeigten Bloch und Kollegen bei 3 von 9 der jungen Patienten eine Verbesserung um mindestens $30 \%$ in der Schwere der depressiven Symptomatik, wobei auch hier unerwünschte Nebenwirkungen auftraten (u.a. Kopfschmerzen, manische Episoden) [67]. Da noch zu wenige Wirksamkeitsnachweise bei Jugendlichen vorliegen, kann das Verfahren nicht empfohlen werden.

\section{Suizidalität}

\section{$\nabla$}

Die schwerwiegendste Komplikation einer depressiven Störung bei Jugendlichen ist das Auftreten suizidaler Tendenzen. Nach Angaben der AACAP berichten bis zu $60 \%$ der depressiven Jugendlichen von suizidalen Gedanken und ca. $30 \%$ haben bereits einen oder mehrere Suizidversuche unternommen [44]. Die größte Gefährdung für Suizide weisen Betroffene im mittleren Jugendalter auf, was mit ihrer kognitiven und emotionalen Entwicklungsstufe zusammenhängt. Jugendliche in diesem Alter leiden sehr häufig unter starker Hoffnungslosigkeit, die einen bedeutenden Risikofaktor für suizidales Verhalten darstellt. Beim Vorliegen einer akuten Suizidgefahr ist in jedem Fall eine stationäre Therapie einzuleiten [1]. In der Praxis sollten mögliche suizidale Gedanken gleich im 1. Gespräch exploriert werden, wobei das Hauptaugenmerk des Untersuchers auf den subjektiven Leidensdruck, die Schwingungsfähigkeit und eine mögliche Agitiert-

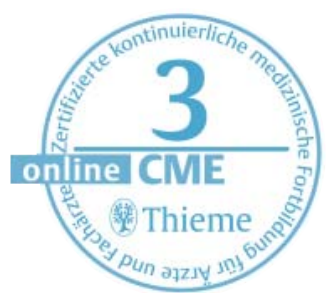


Tab. 8 Erkennen suizidaler Tendenzen bei Jugendlichen (nach [69]).

\begin{tabular}{|c|c|}
\hline Psychopathologie & $\begin{array}{l}\text { - } \text { starke Grübelneigung } \\
\text { - Hoffnungslosigkeit } \\
\text { - psychotische Symptome } \\
\text { - } \text { psychomotorische Unruhe/Agitiertheit } \\
\text { - } \text { plötzliche Stimmungsänderungen } \\
\text { - } \quad \text { schwere Depressionssymptome }\end{array}$ \\
\hline Verhalten & $\begin{array}{l}\text { - } \text { starke Beschäftigung mit dem Thema Tod } \\
\text { - Substanzmissbrauch } \\
\text { - Verwahrlosungstendenzen } \\
\text { - Vernachlässigung von Pflichten } \\
\text { - } \text { Regeln von privaten Angelegenheiten } \\
\text { - } \quad \text { untypische Verhaltensveränderungen }\end{array}$ \\
\hline Interaktion & $\begin{array}{l}\text { - } \text { Abschied nehmen von Angehörigen und Freunden } \\
\text { - } \quad \text { sozialer Rückzug } \\
\text { - Verschenken wichtiger Gegenstände }\end{array}$ \\
\hline
\end{tabular}

heit zu legen ist. Als protektive Faktoren sehen Grube, von Klitzing und Kroll [68]

- eine gute soziale Einbindung und tragfähige Beziehungen zu mindestens einer Bezugsperson,

- eine vorhandene Absprachefähigkeit,

- das Vorliegen von Zukunftsplänen und

- einen guten Zugang zu den eigenen Emotionen.

Neben einem Gespräch mit den Betroffenen und den Eltern können mögliche suizidale Tendenzen auch aus der Interaktion mit dem Jugendlichen oder seinem Verhalten geschlossen werden (॰ Tab.8).

Risikofaktoren für suizidales Verhalten

- Suizidversuche in der Vorgeschichte

- aggressives und/oder impulsives Verhalten

- Traumatisierungen (sexueller Missbrauch, Gewalt)

- Auftreten akuter Stressoren (soziale Isolation, Konflikte)

- Zugang zu Waffen

- komorbide psychische Störungen (z. B. Störungen des Sozialverhaltens, emotional-instabile Persönlichkeitsstörung, Substanzmissbrauch)

- Suizide oder Suizidversuche innerhalb der Familie

- konkrete Suizidplanung (z. B. Abschiedsbriefe)

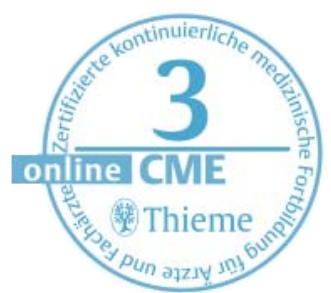

Erhöhen Psychopharmaka die Suizidalität bei Jugendlichen? Neben den psychosozialen Risikofaktoren gibt es Hinweise, dass auch die Einnahme bestimmter antidepressiver Medikamente die Suizidalität von Jugendlichen erhöhen kann. In den letzten Jahren wurde v. a. nach einer psychopharmakologischen Therapie mit SSRI ein Anstieg der suizidalen Gedanken beobachtet, was die Gabe von Antidepressiva bei Jugendlichen immer wieder infrage stellte. 2004 sprach die amerikanische Zulassungsbehörde FDA sogar eine sog. Black-Box-Warnung aus, was in den Folgejahren zu einer Reduktion der Verschreibungen von
SSRI an Jugendliche führte. Hammad, Laughren und Racoosin zeigten in ihrer Übersichtsarbeit eine Steigerung des Suizidrisikos bei Jugendlichen nach der Einnahme von Antidepressiva um durchschnittlich 1,66; der geringste Anstieg wurde nach einer Behandlung mit Fluoxetin verzeichnet $(1,53)$ [70]. Gegenüber Placebo stieg die Suizidgefahr um das Doppelte (2\% nach Einnahme eines Placebos vs. $4 \%$ nach Einnahme von Antidepressiva). Gibbons und Kollegen fanden in ihrer aktuellen Metaanalyse dagegen keinen Anstieg der Suizidalität nach der Einnahme von Fluoxetin und Venlafaxin bei Jugendlichen; bei Erwachsenen führte die Einnahme sogar zu einer Reduktion der suizidalen Tendenzen [71]. Eine Erklärung für diese Ergebnisse ist in einer Altersabhängigkeit von Suizidalität zu suchen: Das Risiko für das Auftreten von suizidalen Gedanken und Verhaltensweisen ist bei jungen Erwachsenen bis 18 Jahren gegenüber Älteren deutlich erhöht [47], wobei v. a. die ersten 4 Wochen ab Behandlungsbeginn ein besonders hohes Risiko bergen.

Zusammenfassend weisen neuere Studien darauf hin, dass auch bei Jugendlichen ohne eine psychopharmakologische Behandlung ein vergleichbar hohes Suizidrisiko vorliegt, sodass gerade in der ersten Zeit eine engmaschige Begleitung der Jugendlichen unabhängig von der Art der Behandlung sinnvoll ist.

Tipp für die Praxis

Neben einer medikamentösen Therapie und einer Betreuung durch einen Kinder- und Jugendpsychiater sollte immer eine psychotherapeutische Behandlung erfolgen, die zu einer Senkung der suizidalen Tendenzen beitragen kann [72].

\section{Tipp für die Praxis \\ Durch die Einnahme von SSRI wird bei Kindern und Jugendlichen, anders als bei Erwachsenen, häufi- ger eine vermehrte Aktivierung ausgelöst (Beha- vioral Toxicity), die Auswirkungen auf suizidale Gedanken und Verhalten haben kann. Daher sollte besonders in der Anfangsphase der Medikamen- tengabe (ca. 4 Wochen) eine engmaschige thera- peutische Betreuung der Patienten mit Erfragen suizidaler Gedanken und Tendenzen erfolgen.}

\section{Neue Entwicklungen in der Therapie depressiver Störungen bei Jugendlichen} $\nabla$

In der Therapie depressiver Störungen bei Erwachsenen nahm in den letzten 10 - 15 Jahren v.a. die Forschung innerhalb der sog. 3. Welle der Verhaltenstherapie zu und es zeigten sich damit gute Behandlungserfolge. $\mathrm{Zu}$ den effektiven neuen Therapieansätzen bei Erwachsenen zählen v.a. die 
- achtsamkeitsbasierte kognitive Therapie (MBCT) [73]

- emotionsfokussierte Therapie [74]

- Mindfulness-Based-Stress-Reduction [75]

- Akzeptanz- und Commitment-Therapie (ACT) [76]

- dialektisch-behaviorale Therapie (DBT) [77]

In Bezug auf die Effektivität der oben genannten Verfahren bei Jugendlichen steht die Forschung derzeit noch am Anfang; es gibt aber erste Hinweise auf eine Wirksamkeit auch in dieser Altersgruppe [78]. Neben den genannten Verfahren ist aktuell auch eine Übersichtsarbeit zum Einsatz der Schematherapie bei depressiven Kindern und Jugendlichen in Vorbereitung [79].

ACT. Hayes, Bach und Boyd untersuchten in einer Pilotstudie mit 30 depressiven Jugendlichen die Wirksamkeit der ACT in einem ambulanten Setting [80]. Es zeigte sich eine deutliche Überlegenheit dieses Verfahrens gegenüber der normalen Therapie, die auch bei einem Follow-up nach 3 Monaten noch nachgewiesen werden konnte. Ziel der ACT in der Behandlung von Jugendlichen ist es, diese in ihrer Gesamtheit wahrzunehmen und mit Einbezug biografischer und situativer Informationen ein Verständnis und eine Veränderung herbeizuführen. Ziel ist nicht die Veränderung der Inhalte von Kognitionen, sondern die Jugendlichen sollen lernen, ihre depressiven Gedanken als solche zu erkennen und diese anzunehmen, während sie im Hier und Jetzt an ihren Zielen arbeiten. Dazu nutzt die ACT neben verhaltenstherapeutischen Techniken v.a. achtsamkeitsbasierte Interventionen und Interventionen zur Werteklärung.

Zentrale Prozesse der ACT [80]

- Akzeptanz der eigenen Gedanken und Gefühle

- bewusste Distanzierung von depressiven Gedanken

- Leben im Hier und Jetzt

- sich selbst als veränderlich wahrnehmen

- Klärung und Bewusstmachung eigener Ziele und Werte

- eigenes Verhalten bewusst steuern und Eigenverantwortung übernehmen

Behavioral Activation. Neben der ACT scheint auch das Vorgehen der Behavioral Activation (BA) bei Jugendlichen mit Depressionen wirksam zu sein [81]. Bei diesem Verfahren stehen weniger die Kognitionen, als vielmehr das Verhalten im Vordergrund: Die Jugendlichen lernen mithilfe angewandter Verhaltensanalysen ihr dysfunktionales Verhalten (Rückzug, Passivität, Vermeidung) kennen und sollen dieses durch die Therapie verändern, was in der Folge die soziale Einbindung und die Selbstwirksamkeitsgefühle der Jugendlichen positiv beeinflusst. Vorteile des Verfahrens liegen in der verhaltensorientierten Herangehensweise. Diese kann auch bei Kindern und Jugendlichen eingesetzt werden, die aufgrund des Alters und ihrer Entwicklungsstufe noch wenig Zugang zu ihren Kognitionen haben.

\section{Kernaussagen}

Aus den Leitlinien der DGKJP $[1,6]$ lassen sich folgende Hinweise für die Praxis ableiten:

- Für die Diagnosestellung einer depressiven Episode bei Jugendlichen können die bei Erwachsenen gültigen Kriterien der ICD-10 (F3) herangezogen werden, auch wenn besondere Symptome für jede Altersgruppe miteinbezogen werden sollen. Zur Einteilung des Schweregrads werden immer auch das jeweilige soziale und psychische Funktionsniveau herangezogen.

- Neben dem Einsatz von diagnostischen Verfahren geben auch fremdanamnestische Daten von Eltern und anderen Bezugspersonen und auch Verhaltensbeobachtungen Hinweise auf die Symptomatik.

- Bei Kindern und Jugendlichen sollten vor einer Behandlung mögliche organische Ursachen der Symptome abgeklärt werden. Differenzialdiagnostisch ist v. a. der Ausschluss einer bipolaren Störung notwendig, da hier zum Teil eine andere Behandlung anzuwenden ist.

- Bei Kindern und Jugendlichen empfiehlt sich eine Behandlung im ambulanten Setting. Eine stationäre Therapie sollte v. a. bei akuter Suizidalität und psychotischen Symptomen eingeleitet werden.

- Psychotherapie stellt in der Behandlung depressiver Störungen bei Jugendlichen die Therapie der 1. Wahl dar. Vor allem die Kognitive Verhaltenstherapie zeigt in vielen Studien eine hohe Wirksamkeit.

- Bei schweren Verläufen oder einem Nichtansprechen auf eine Psychotherapie kann ergänzend eine psychopharmakologische Behandlung begonnen werden. Dabei haben sich v. a. selektive Serotonin-Wiederaufnahmehemmer als wirksam erwiesen. Als Mittel der 1. Wahl sollte Fluoxetin gegeben werden, das als einziges Präparat für diese Altersgruppe zugelassen ist und das niedrigste Suizidrisiko in der Phase der Eindosierung aufweist.

- Bei vorliegender Indikation sollten auch Maßnahmen der Jugendhilfe oder andere rehabilitative Maßnahmen angedacht werden.

Interessenkonflikte: Die Autoren geben an, dass kein Interessenkonflikt besteht.

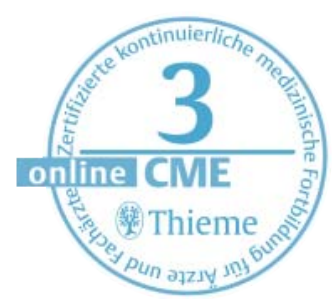


Literatur

1 AWMF. Behandlung von depressiven Störungen bei Kindern und Jugendlichen. AWMF S3 Leitlinie. 2013

2 Wijlaars LPMM, Nazareth I, Petersen I. Trends in depression and antidepressants prescirbing in children and adolescents: a cohort study in The Health Improvement Network (THIN). PLoS ONE 7: e33181. DOI: 10.1371/journal.pone.0033181

3 Hoffmann F, Petermann F, Glaeske G et al. Prevalence and comorbidities in adolescent depression in Germany: an analysis of health insurance data. Zeitschrift für Kinder- und Jugendpsychiatrie und -psychotherapie 2012; 40: 399-404

4 Boyd CP, Gullone E, Konstanski $M$ et al. Prevalence of anxiety and depression in Australian adolescents: comparisons with world-wide-data. J Genetic Psychology: Research and Theory on Human Development 2000; 161: 479-492

5 Dilling H, Mombour W, Schmidt MH. Internationale Klassifikation psychischer Störungen ICD-10 Kapitel V (F). Klinisch-diagnostische Leitlinien. Bern: Hans Huber Verlag; 2008

6 Deutsche Gesellschaft für Kinder- und Jugendpsychiatrie und Psychotherapie. Leitlinien zur Diagnostik und Therapie psychischer Störungen im Säuglings-, Kindes- und Jugendalter. 3 Aufl. Köln: Deutscher Ärzte Verlag; 2007

7 Koplewicz HS. Adolescent Depression. J Child and Adolescent Psychopharmacology 2012; 22: 1

8 Ihle W, Groen G, Walter D et al. Depression. Leitfaden Kinder- und Jugendpsychiatrie. Band 16. Göttingen: Hogrefe Verlag; 2012

9 Unnewehr S, Schneider S, MargrafJ. Kinder-DIPS: Diagnostisches Interview bei psychischen Störungen im Kindes- und Jugendalter. Göttingen: Hogrefe Verlag; 2009

10 Rotter JB, Lah MI, Rafferty JE. Rotter Incomplete Sentences Blank Second Edition Manual. New York: Psychological Corporation; 1992

11 Stiensmeier-Pelster J, Schürmann M, Duda K. DIKJ: Depressionsinventar für Kinder und Jugendliche. Göttingen: Hogrefe Verlag; 2000

12 Rossmann P. Depressionstest für Kinder (DTK). 2 Aufl. Bern: Hogrefe Verlag; 2005

13 Hautzinger M, Keller F, Kühner C. BDI-II: Becks Depressions-Inventar Revision. Harcourt Test Services; 2006

14 Keller F, Grieb J, Kölch M, Spröber M. Children's Depression Rating Scale: Revised by E. O. Poznanski \& H. B. Mokros: Deutsche Version. Göttingen: Hogrefe Verlag; 2012

15 Hamilton MA. A rating scale for depression. J Neurology, Neurosurg Psychiatry 1960; 23: 56-62

16 Petermann F, Petermann U. HAWIK-IV. 3 Aufl. Bern: Huber; 2010

17 Shashi K, Bhatia MD, Subhash D et al. Childhood and Adolescent Depression. Am Acad Family Physicians 2007; 75: 73-80

18 Birmaher $B$, Brent $D$. Practice parameters for the treatment of children and adolescents with depressive disorders. J Am Acad Child Adolescent Psychiatry 2007; 46: 1503-1526

19 Hyde JS, Mezulis AH, Abramson LY. The ABC of depression: Integrating affective, biological, and cognitive models to explain the emergence of gender difference in depression. Psychological Rev 2008; 115: 291 313

20 Schulte-Körne G, Schiller Y. Wirksamkeit universeller und selektiver Pävention von Depression im Kindes- und Jugendalter. Ein systematischer Review. Z Kinder- und Jugendpsychiatrie und Psychotherapie 2012; 40: 385-397

21 Gilham JE, Reivich KJ, Brunwasser SM et al. Evaluation of a group cognitive-behavioral depression prevention programe for young adolescents: a randomized effectivness trial. J Clin Child \& Adolescent Psychology 2012; 41: 621-639

22 Horowitz JL, Garber J, Ciesla JA et al. Prevention of depressive symptoms in adolescents: a randomized trialof cognitive-behavioral and interpersonal prevention program. J Consulting \& Clinical Psychology 2007; 75: $693-706$

23 Pössel P, Adelson JL, Hautzinger M. A randomized trial to evaluate the course of effects of a program to prevent adolescent depressive symptoms over 12 months. Behav Res Therapy 2011; 49: 838-851

24 Stallard $P$, Sayal K, Phillips R et al. Classroom based cognitive behavioural therapy in reducing symptoms of depression in high risk adolescents: pragmatic cluster randomised controlled trial. Br Med J 2012; 345: e6058. DOI: 10.1136/bmj.e6058

25 Pössel P, Martin NC, Garber J et al. A randomized controlled trial comparing a cognitive-behavioral to a non-specific program for the pre- vention of depression in adolescents. Assoc Child Adolescent Mental Health 2012; 121: 838-851. DOI: 10.1037/a0028175

26 Merry SN. Preventing depression in adolescents. Br Med J 2012; 345: e6720. DOI: 10.1136/bmj.e6720

27 Merry SN, Stasiak K, Shepherd $M$ et al. The effectiveness of SPARX, a computerised self help intervention for adolescents seeking help for depression: randomised controlled non-inferiority trial. $\mathrm{Br}$ Med J 2012; 344: e2598. DOI: 10.1136/bmj.e2598

28 Caporino NE, Karver MS. The acceptability of treatments for depression to a community sample of adolescent girls. J Adolescence 2012; 35: $1237-1245$

29 Groen G, Petermann F. Kognitive Verhaltenstherapie bei Depressionen im Kindes- und Jugendalter. Vorgehen, Wirksamkeit und Weiterentwicklungen. Z Kinder- und Jugendpsychiatrie und Psychotherapie 2012; 40: $373-384$

30 Curry JF. Specific psychotherapies for children and adolescent depression. Soc Biological Psychiatry 2001; 49: 1091 - 1100

31 Erford BT, Erford BM, Lattanzi G et al. Counseling outcomes from 1990 to 2008 for school-age youth with depression: a meta-analysis. J Counseling \& Development 2011; 89: 439-457

32 Abel UA. Konzeption und Evaluation einer kognitiv-verhaltenstherapeutischen Gruppentherapie für depressive Jugendliche. unveröffentlichte Dissertation. Tübingen: Eberhard Karls Universität; 2010

33 Ströber N, Straub J, Fegert JM, Kölch M. Depression im Jugendalter: MICHI - Manual für die Gruppentherapie. Weinheim: Beltz; 2012

34 Straub J, Kölch M, Plener P et al. MICHI: A brief cognitive-behavioural group therapy for adolescents with depression. A pilot study of feasibility in an inpatient setting. Child Adolescent Mental Health 2012, [accepted]

35 Mufson L, Pollack KD, Wickramaratne P et al. A randomized effectiveness trial of interpersonal psychotherapy for depressed adolescents. Arch General Psychiatry 2004; 61: 577-584

36 Krysta K, Kryzstanek M, Janas-Kozik M et al. Bright light therapy in the treatment of child and adolescent depression, antepartum depression, and eating disorders. J Neural Transmission 2012; 119: 1167-1172

37 Bachmann M, Bachmann C, Rief $C$ et al. Wirksamkeit psychiatrischer und psychotherapeutischer Behandlungen bei psychischen Störungen von Kindern und Jugendlichen. Eine systematische Auswertung der Ergebnisse von Metaanalysen und Reviews. Teil II: Angststörungen und depressive Störungen. Z Kinder- und Jugendpsychiatrie und Psychotherapie 2008; 36: 309-320

38 Mehler-Wex C, Kölch M. Depressive Störungen im Kindes- und Jugendalter. Dt Ärzteblatt 2008; 105: 149-155

39 Merry SN, Hetrick SE, Cox GR et al. Psychological and educational interventions for preventing depression in children and adolescents. Cochrane Database of Systematic Reviews 2011, (12): Art. No.: CD 003380. DOI: $10.1002 / 14651858 . C D 003380 . p u b 3$

40 Hoek W, Schuurmans J, Koot HM et al. Effects of internet-based guided self-help problem-solving therapy for adolescents with depression and anxiety: a randomized controlled trial. PLos ONE 7: e43485. DOI: 10.1371/journal.pone.e0043485

41 Kratochvil C, Emslie G, Silva S et al. Acute time to respond in the Treatment of Adolescent with Depression Study. J Am Acad Child and Adolescent Psychiatry 2006; 45: 1412 - 1418

42 Cox GR, Callahan P, Churchill R et al. Psychological therapies versus antidepressant medication, alone and in combination for depression in children and adolescents. Cochrane Database of Systematic Reviews 2012, (11): Art. No.: CD008324. DOI: 10.1002/14651858.CD008324. pub2

43 Curry JF, Rhode P, Simons A et al. Predictors and moderators of acute outcome in the Treatment of Adolescents with Depression Study. J Am Acad Child and Adolescent Psychiatry 2006; 45: 1472 - 1493

44 Hetrick SE, McKenzie JE, Cox GR et al. Newer generation of antidepressants for depressive disorders in children and adolescents. Cochrane Database of Systematic Reviews 2012, (11): Art. No.: CD004851. DOI: 10.1002/14651858.CD004851.pub3

45 American Academy of Child and Adolescet Psychiatry. Practice Parameter for the Assessment and Treatment of Child and Adolescents Depressive Disorder. J Am Acad Child and Adolescent Psychiatry 2007; 46: $1503-1526$

46 NICE 28-2005. National Institute of Clinical Health and Excellence. Depression in children and young people. Identification and management in primary, community, and secondary care. NICE; 2005: 28

47 Plener PL, Fegert JM, Kölch M. Psychopharmakologische Behandlung von Depressionen im Kindes- und Jugendalter. Entwicklungen und 
Standards seit der „Black Box“-Warnung. Z Kinder- und Jugendpsychiatrie und Psychotherapie 2012; 40: 365-371

48 Hofmann F, Glaeske G, Petermann F et al. Outpatient treatment with in German adolescents with depression: An analysis of nationwide health insurance data. Pharmacoepidemiology Drug Safety 2012; 21: 972-979

49 Goodyer I, Dubicka B, Wilkinson P et al. Selective serotonine reuptake inhibitors (SSRI) and routine specialist care with and without cognitive behavior therapy in adolescents with major depression: randomised controlled trial. Br Med J 2007; 335: $142-150$

50 The Treatment of Adolescents Depression Study Team. Fluoxetine, cognitive behavioral therapy and their combination for adolescents with depression. J Am Med Assoc 2004; 292: 807-820

51 Wagner KD, Robb AS, Findling RL et al. A randomized-placebo-controlled trial with citalopram for the treatment of major depression in children and adolescents. Am J Psychiatry 2004; 161: 1079-1083

52 Wagner KD, Ambrosini P, Rynn M et al. Efficacy of sertraline in the treatment of children and adolescents with major depressive disorder two randomized controlled trials. J Am Med Assoc 2003; 290: 1033 1041

53 FDA: Review and evaluation of clinical data. www.fda.gov/ohrms/dockets/ac/04/briefing/2004-4065b110-TAB08-Hammads-Review.pdf

54 Holtmann M, Bölte S, Wöckel L et al. Antidepressive Therapie bei Kindern und Jugendlichen. Anwendung und Stellenwert der Selektiven-Serotonin-Wiederaufnahmehemmer. Dt Ärzteblatt 2005; 102: 976 - 978

55 Brent D, Emslie G, Clark G. Switching to another SSRI or venlafaxine with or without cognitive behavioral therapy for adolescents with SSRI-resistant depression: the TORDIA randomized controlled trial. J Am Med Assoc 2008; 299: 901 -913

56 Daviss WB, Bentivoglio $P$, Racusin $R$ et al. Bupropion sustained release in adolescents with comorbid attention-deficit/hyperactivity disorder and depression. J Am Assoc Child Adolescent Psychiatry 2004; 40 $307-314$

57 Pathak S, Johns ES, Kowatch RA. Adjunctive quetiapine for treatmentresistant adolescent major depressive disorders: a case series. J Child Adolescent Psychopharmacology 2005; 15: 696-702

58 Hazell P, O'Connell D, Heathcote A et al. Tricyclic drugs for depression in children and adolescents. Cochrane Database Systematic Review 2002 (2): Art. No.: CD002317. DOI: 10.1002/14651858.CD002317

59 Emslie GJ, Findling RL, Yeung PP et al. Efficacy and safety of venlafaxine ER in children and adolescents with major depression disorder. Poster presented at the 157th Annual Meeting of the American Psychiatric Association. New York: May 1-6 2004

60 Mandoki MW, Tapia MR, Tapia MA et al. Venlafaxine in the treatment of children and adolescents with major depression. Psychopharmacological Bull 1997; 33: 149-154

61 Prakash A, Lobo E, Kratochvil CJ et al. An Open-Label Safety and Pharmacokinetics Study of Duloxetine in Pediatric Patients with Major Depression. J Child Adolescent Psychopharmacology 2012; 22: 48-55

62 Ishihara $K$, Sasa $M$. Mechanism underlying the therapeutic effects of electroconvulsive therapy (ECT) on depression. Japanese J Pharmacology 1999; 80: 185-189
63 Ghaziuddin N, Kutcher SP, Knapp P et al. Practice parameter for use of electroconvulsive therapy with adolescents. J Am Acad Child Adolscent Psychiatry 2004; 43: 1521 - 1539

64 Strober M, Rao U, DeAntonio $M$ et al. Effects of electroconvulsive therapy in adolescents severe endogenous depression resistant to pharmacotherapy. Biological Psychiatry 1998; 43: 335 - 338

65 American Academy of Child and Adolescent Psychiatry. Practice Parameter for Use of Electroconvulsive Therapy with Adolescents. J Am Acad Child Adolescent Psychiatry 2004; 43: 1521 - 1539

66 Avery DH, Holtzheimer PE III, Fawaz W et al. A controlled study of transcranial magnetic stimulation in medication-resistant major depression. Biol Psychiatry 2006; 59: 187-194

67 Bloch Y, Grisaru N, Harel EV et al. Repetitive transcranial magnetic stimulation in the treatment of depression in adolescents: an open-label study. J ECT 2008; 24: 156-159

68 Grube M, von Klitzing K, Kroll M. Depressionen im Kindes- und Jugendalter. Pädiatrische Praxis 2011; 77: 607-621

69 Essau CA, Petermann U. Depression. In: Petermann F Hrsg. Lehrbuch der Klinischen Kinderpsychologie und -psychotherapie. 4 Aufl. Göttingen: Hogrefe Verlag; 2000: 291 - 322

70 Hammad TA, Laughren T, Racoosin J. Suicidality in pediatric patients treated with antidepressantdrugs. Arch General Psychiatry 2006; 63: $332-339$

71 Gibbons RD, Brown H, Hur K et al. Suicidal thoughts and behaviour with antidepressant treatment. Arch General Psychiatry. DOI: 10.1001/ archgenpsychiatry.2011.2048

72 Dubicka B, Elvins R, Roberts C et al. Combined treatment with cognitive behavioural therapy in adolescent depression: a meta-analysis. $\mathrm{Br}$ Psychiatry 2010; 197: 433-440

73 Segal ZV, Williams JMG, Teasdale JD. Die achtsamkeitsbasierte Kognitive Therapie der Depressionen. Ein neuer Ansatz zur Rückfallprävention. Tübingen: dgvt; 2002

74 Greenberg LS. Emotionsfokussierte Therapie: Lernen mit den eigenen Gefühlen umzugehen. Tübingen: dgvt-Verlag; 2002

75 Kabat-Zinn J. Full catastrophe living: Using the wisdom of your body and mind to face stress, pain and illness. New York: Dell; 1990

76 Hayes SC, Luoma J, Walser RD. ACT-Training: Handbuch der Acceptance \& Commitment Therapie. Paderborn: Junfermann; 2008

77 Linehan M. Dialektisch-behaviorale Therapie der Borderline-Persönlichkeitsstörung. München: CIP-Medien; 1996

78 Greco L, Hayes SC. Akzeptanz und Achtsamkeit in der Kinder- und Jugendlichenpsychotherapie. Weinheim: Beltz; 2011

79 Loose C, Graaf P, Zarbock G. Schematherapie mit Kindern und Jugendlichen. Weinheim: Beltz [in Vorbereitung]

80 Hayes SC, Bach PA, Boyd CP. Psychological treatment for adolescent depression: Perspectives on the past, present, and future. Behaviour Change 2010; 27: $1-18$

81 McCauley E, Schloredt K, Gudmundsen G et al. Expanding behavioral activation to depressed adolescents: lessons learned in treatment development. Cognitive Behavioral Practice 2011; 18: 371 - 383 


\section{CME-Fragen Depressive Störungen bei Jugendlichen: Diagnostik und Therapie}

1 Welche der folgenden Symptome stehen bei depressiven Kindern im späten Kindesalter im Vordergrund?

Selbstverletzung

Spielunlust

geringes Selbstwertgefühl

Sorgen und Ängste

Ess- und Schlafprobleme

2 Welches der folgenden Symptome tritt v. a. bei depressiven Kindern im Vorschulalter auf?

starke Müdigkeit

Suizidgedanken

somatische Beschwerden

Langeweile

Selbstverletzung

3

Welches der folgenden Symptome kann auf das Vorliegen einer depressiven Episode im Rahmen einer bipolaren Störung hinweisen?

A akuter Beginn

B leichte Ausprägung der depressiven Symptomatik

C vorwiegend starke Niedergeschlagenheit

D somatische Beschwerden

E schleichender Beginn

Welches der folgenden Merkmale gilt nicht als Risikofaktor einer depressiven Erkrankung im Kindes- und Jugendalter? emotional-instabile Persönlichkeitszüge weibliches Geschlecht

hohe Selbstwirksamkeitserwartung

Vorliegen von Depressionen innerhalb der Familie

familiäre Konflikte

Welche der folgenden Aussagen zur Diagnostik bei Kindern und Jugendlichen mit depressiver Störung trifft nicht zu? Neben strukturierten und halbstrukturierten Interviews werden auch störungsspezifische Selbstbeurteilungsverfahren angewendet.

B Fremdanamnestische Angaben durch Eltern, Lehrer und anderen Bezugspersonen sollten immer eingeholt werden.

C Eine Leitungsdiagnostik dient dem Ausschluss von möglicher symptomauslösender Über- oder Unterforderung in der Schule.

D Verhaltensbeobachtungen sind relevant zur Beurteilung der Ausprägung und des Verlaufs der Symptomatik.

E Unauffällige Testergebnisse können das Vorliegen einer depressiven Störung ausschließen.

Welche Aussage zur Psychopharmakotherapie trifft nicht zu?

Bei mittelgradigen bis schweren depressiven Episoden sollte eine ergänzende psychopharmakologische Behandlung angedacht werden.

B In der Behandlung von Depressionen bei Kindern und Jugendlichen empfiehlt sich der Einsatz von SSRI.
C Eine psychopharmakologische Behandlung sollte v. a. in den ersten 4 Wochen intensiv psychiatrisch und psychotherapeutisch begleitet werden.

D Sowohl die Betroffenen als auch die Erziehungsberechtigten müssen über die Wirkungen und Nebenwirkungen der Medikamente informiert werden.

E In der Behandlung von Kindern haben sich auch trizyklische Antidepressiva als wirksam erwiesen.

7 Welches SSRI ist in Europa in der Behandlung von Kindern ab 8 Jahren zugelassen?

Sertralin

Fluoxetin

Paroxetin

Citalopram

alle genannten Medikamente

8 Welche Erkrankung gehört nicht zu den wichtigsten organischen Differentialdiagnosen der depressiven Störung bei Jugendlichen?

A Diabetes mellitus

B Magenulcus

C Hypothyreose

D Anämie

E Grippale Infekte

A Der Aufbau einer vertrauensvollen therapeutischen Beziehung ist bei depressiven Patienten zu Beginn der Behandlung nicht möglich.

B Die Effektivität des Einbezugs von Eltern in die Therapie bei Jugendlichen ist nicht geklärt.

c Die kognitive Verhaltenstherapie gilt als Therapie der 1. Wahl.

D Neben der einzeltherapeutischen Behandlung sind auch gruppentherapeutische Angebote effektiv.

E Auch für die Interpersonale Therapie (IPT) besteht eine Effektivität für die Behandlung von Depressionen bei Jugendlichen.

Welche der folgenden Aussagen hinsichtlich der Suizidalität bei depressiven Jugendlichen trifft nicht zu?

A Als protektive Faktoren gelten u. a. eine gute soziale Einbindung und tragfähige Beziehungen zu mindestens einer Bezugsperson.

B Interaktionen mit dem Jugendlichen oder Gespräche geben selten Hinweise auf suizidale Tendenzen.

c Beim Vorliegen akuter Suizidalität ist eine stationäre Therapie einzuleiten.

D Betroffene im mittleren Jugendalter weisen die höchste Gefährdung für Suizide auf.

E Starke Hoffnungslosigkeit ist ein bedeutender Risikofaktor für suizidales Verhalten. 